\title{
FRW cosmological perturbations in massive bigravity
}

\author{
D. Comelli, ${ }^{1,2, *}$ M. Crisostomi, ${ }^{3,4,5, \dagger}$ and L. Pilo ${ }^{4,6, *}$ \\ ${ }^{1}$ INFN, Sezione di Ferrara, I-35131 Ferrara, Italy \\ ${ }^{2}$ CERN, Theory Division, 1211 Geneva, Switzerland \\ ${ }^{3}$ School of Physics and Astronomy, University of Nottingham, Nottingham NG7 2RD, United Kingdom \\ ${ }^{4}$ INFN, Laboratori Nazionali del Gran Sasso, I-67010 Assergi, Italy \\ ${ }^{5}$ Université Paris Diderot-Paris 7, F-75205 Paris, France \\ ${ }^{6}$ Dipartimento di Fisica, Università di L'Aquila, I-67010L'Aquila, Italy \\ (Received 11 June 2014; published 2 October 2014)
}

\begin{abstract}
Cosmological perturbations of Friedmann-Robertson-Walker solutions in ghost free massive bigravity, including also a second matter sector, are studied in detail. At early time, we find that subhorizon exponential instabilities are unavoidable and they lead to a premature departure from the perturbative regime of cosmological perturbations.
\end{abstract}

DOI: 10.1103/PhysRevD.90.084003

PACS numbers: 04.50.Kd, 98.80.-k

\section{INTRODUCTION}

Dark energy is the dominant component of our Universe; if future observations will establish that its equation of state differs from the one of a cosmological constant contribution, then we have a case for modifying general relativity (GR) at large distances and massive gravity can be a compelling candidate. Great effort was devoted to extend at the nonlinear level [1,2] the seminal work of Fierz-Pauli [3] and recently a Boulware-Deser (BD) ghost free theory was found [4,5]. Unfortunately, cosmological solutions of the ghost free De Rham-Gabadaze-Tolley theory are rather problematic: spatially flat homogenous FriedmannRobertson-Walker (FRW) solutions simply do not exist [6] and even allowing for open FRW solutions [7], strong coupling [8] and ghostlike instabilities [9,10] develop. In addition, the cutoff of the theory is rather low [11], namely $\Lambda_{3}=\left(m^{2} M_{\mathrm{Pl}}\right)^{1 / 3}$. For a recent review see $[12,13]$.

A possible way out is giving up Lorentz invariance which requires only rotational invariance [14-16]. Within the general class of theories that propagate 5 degrees of freedom $(\mathrm{DoF})$ found in $[17,18]$, in the Lorentz breaking case most of the theories have a much safer cutoff $\Lambda_{2}=$ $\left(m M_{\mathrm{Pl}}\right)^{1 / 2} \gg \Lambda_{3}$ and also avoid all of the phenomenological difficulties mentioned above, including troubles with cosmology [19]. Another option is to promote the nondynamical metric entering in the construction of massive gravity theory to a dynamical one $[20,21]$ entering in the realm of bigravity originally introduced by Isham, Salam, and Strathdee [22].

In the bigravity formulation, FRW homogenous solutions do exist [23-25]; however, cosmological perturbations, for modes inside the horizon, start to grow too early and too fast when compared with GR, and as a result the

\footnotetext{
*comelli@fe.infn.it

marco.crisostomi@aquila.infn.it

†luigi.pilo@aquila.infn.it
}

linear regime becomes problematic already during the radiation and matter era [26]. The reason of such peculiar behavior of the scalar perturbations could be naively traced back to the FRW background solution that is controlled by the parameter $\xi$ (the ratio of the conformal factors of the two metrics) and to the absence of matter coupled to the second metric whose pressure could support inside horizon gravitational perturbations.

In the presence of only ordinary matter, coupled with the first metric, only small values of the parameter $\xi$ give an acceptable early time cosmology. The introduction of the second matter component provides other consistent background solutions where the values of $\xi$ can be also of order 1 and, at the same time, provides the necessary pressure support to infall perturbations.

In this paper we will extend our previous analysis to the case where an additional matter sector is minimally coupled to the second metric. Though we do not consider the problem, the second matter sector could be also relevant for dark matter $[27,28]$.

The outline of the paper is the following: In Sec. II we review the bigravity formulation of massive gravity and the extension to the case where a second matter sector is present. In Sec. III we study FRW solutions, and cosmological perturbations are analyzed in Sec. IV.

\section{MASSIVE GRAVITY AND BIGRAVITY}

Any modification of GR that turns a massless graviton into a massive one calls for additional DoF. Basically, GR is deformed by a scalar function $V$ built from the physical metric $g$ that couples with matter and a second metric $\tilde{g}$. Besides the phenomenological issue, dealing with a nondynamical metric is rather awkward; in this context it is natural to promote the second metric to a fully dynamical field; see for instance [29]. Thanks to $\tilde{g}$, it is possible to build nontrivial diffeomorphism invariant terms without derivatives of the metric. Expanding the metric around a 
fiducial background, such terms lead precisely to the mass term for the graviton. Consider the action

$$
\begin{aligned}
S= & \int d^{4} x\left\{\sqrt{\tilde{g}}\left[\kappa M_{\mathrm{pl}}^{2} \tilde{R}+L_{\tilde{\mathrm{M}}}\right]\right. \\
& \left.+\sqrt{g}\left[M_{\mathrm{pl}}^{2}\left(R-2 m^{2} V\right)+L_{\mathrm{M}}\right]\right\},
\end{aligned}
$$

where $R$ and $\tilde{R}$ are the corresponding Ricci scalars and the deforming potential $V$ is a scalar function of the tensor $X_{\nu}^{\mu}=g^{\mu \alpha} \tilde{g}_{\alpha \nu}$. Ordinary matter is minimally coupled with $g$ and is described by $L_{\mathrm{M}}$. In order try to cope with the instabilities found in [26], we shall introduce a second matter sector that couples minimally with $\tilde{g}$ and it is described by $L_{\tilde{\mathrm{M}}}$. The constant $\kappa$ controls the relative size of the strength of gravitational interactions in the two sectors, while $m$ sets the scale of the graviton mass. In particular, in the limit $\kappa \rightarrow \infty$, the second metric gets frozen to a prescribed background value. Removing the second matter sector one recovers the previously studied bigravity theories; see for instance [30].

The modified Einstein equations can be written as ${ }^{1}$

$$
\begin{aligned}
E_{\nu}^{\mu}+Q_{1 \nu}^{\mu} & =\frac{1}{2 M_{\mathrm{pl}}^{2}} T_{\nu}^{\mu}, \\
\kappa \tilde{E}_{\nu}^{\mu}+Q_{2 \nu}^{\mu} & =\frac{1}{2 M_{\mathrm{pl}}^{2}} \tilde{T}_{\nu}^{\mu},
\end{aligned}
$$

where we have defined $Q_{1}$ and $Q_{2}$ as effective energymomentum tensors induced by the interaction term. The ghost free potential $[2,4]^{2} V$ is a special scalar function of $Y_{\nu}^{\mu}=(\sqrt{X})_{\nu}^{\mu}$ given by

$$
\begin{aligned}
V & =\sum_{n=0}^{4} a_{n} V_{n}, \quad n=0 \ldots 4, \quad \tau_{n}=\operatorname{Tr}\left(Y^{n}\right) ; \\
V_{0} & =1 \quad V_{1}=\tau_{1}, \quad V_{2}=\tau_{1}^{2}-\tau_{2}, \\
V_{3} & =\tau_{1}^{3}-3 \tau_{1} \tau_{2}+2 \tau_{3}, \\
V_{4} & =\tau_{1}^{4}-6 \tau_{1}^{2} \tau_{2}+8 \tau_{1} \tau_{3}+3 \tau_{2}^{2}-6 \tau_{4} .
\end{aligned}
$$

In [32] it was shown that in the bimetric formulation the potential $V$ is BD ghost free. We have that

$$
\begin{aligned}
Q_{1 \nu}^{\mu} & =m^{2}\left[V \delta_{\nu}^{\mu}-\left(V^{\prime} Y\right)_{\nu}^{\mu}\right], \\
Q_{2 \nu}^{\mu} & =m^{2} q^{-1 / 2}\left(V^{\prime} Y\right)_{\nu}^{\mu},
\end{aligned}
$$

where $\left(V^{\prime}\right)_{\nu}^{\mu}=\partial V / \partial Y_{\mu}^{\nu}$ and $q=\operatorname{det} X=\operatorname{det}(\tilde{g}) / \operatorname{det}(g)$.

The canonical analysis [32,33] shows that, in general, 7 DoF propagate; around a Minkowski background, 5 can be

\footnotetext{
${ }^{1}$ When not specified, indices of tensors related with $g(\tilde{g})$ are raised or lowered with $g(\tilde{g})$.

${ }^{2}$ A very similar potential having the same form but with $X$ instead of $X^{1 / 2}$ was considered in [31].
}

associated to a massive spin two graviton and the remaining 2 to a massless spin two graviton. We consider only the case where each matter sector is minimally coupled with only its own metric field. Allowing the second metric to couple also with standard matter would result in a violation of the equivalence principle; indeed, it is not possible to locally put both metrics in a Minkowski form.

\section{FRW SOLUTIONS IN MASSIVE BIGRAVITY}

Let us consider FRW background solutions in massive bigravity of the form

$d s^{2}=a^{2}(\tau)\left(-d \tau^{2}+d r^{2}+r^{2} d \Omega^{2}\right)=\bar{g}_{1 \mu \nu} d x^{\mu} d x^{\nu}$,

$\tilde{d} s^{2}=\omega^{2}(\tau)\left[-c^{2}(\tau) d \tau^{2}+d r^{2}+r^{2} d \Omega^{2}\right]=\bar{g}_{2 \mu \nu} d x^{\mu} d x^{\nu}$.

It is convenient to define the standard Hubble parameters for the two metrics and the ratio between the two scale factors

$\mathcal{H}=\frac{d a}{d \tau} \frac{1}{a} \equiv \frac{a^{\prime}}{a}, \quad \mathcal{H}_{\omega} \equiv \frac{\omega^{\prime}}{\omega}=\frac{\xi^{\prime}}{\xi}+\mathcal{H}, \quad \xi \equiv \frac{\omega}{a} ;$

where with ' we always denote the derivation with respect to the conformal time $\tau$. Solutions fall in two branches depending on how the covariant conservation of $Q_{1 / 2}$, enforced by the Bianchi identities is realized. It turns out that the physically interesting case $[23,26]$ is when, as a consequence of the conservation of $Q_{1 / 2}$, we have that

$$
c=\frac{\mathcal{H}_{\omega}}{\mathcal{H}}, \quad \xi^{\prime}=(c-1) \mathcal{H} \xi \quad \text { with } \quad c>0 .
$$

We will not discuss the other branch of solutions where $\xi$ is constant and the effect of gravity modification amounts to a cosmological constant and perturbations are strongly coupled [26], as expected.

The expansion rate follows from the equation

$$
\frac{3 \mathcal{H}^{2}}{a^{2}}=8 \pi G \rho_{1}+m^{2}\left(6 a_{3} \xi^{3}+6 a_{2} \xi^{2}+3 a_{1} \xi+a_{0}\right) .
$$

The presence of the second metric is equivalent, for the first sector, to a gravitational fluid with energy density $\rho_{g}$ given by

$$
\rho_{g}=\frac{m^{2}\left[6 \xi^{2}\left(a_{3} \xi+a_{2}\right)+3 a_{1} \xi+a_{0}\right]}{8 \pi G} ;
$$

with an equation of state $p_{g}=w_{g} \rho_{g}$ of the form

$$
w_{g}=-1-\frac{\left(6 a_{3} \xi^{2}+4 a_{2} \xi+a_{1}\right) \xi^{\prime}}{\mathcal{H}\left[6 \xi^{2}\left(a_{3} \xi+a_{2}\right)+3 a_{1} \xi+a_{0}\right]} .
$$


The conservation of energy-momentum tensor for the two fluids leads to

$\rho_{1}^{\prime}+3 \mathcal{H}\left(\rho_{1}+p_{1}\right)=0, \quad \rho_{2}^{\prime}+3 \mathcal{H}_{\omega}\left(\rho_{2}+p_{2}\right)=0$,

thus for $p_{i}=w_{i} \rho_{i}$ we have $\rho_{1}=\rho_{1}^{\text {in }} a^{-3\left(w_{1}+1\right)}$ and $\rho_{2}=$ $\rho_{2}^{\text {in }} \omega^{-3\left(w_{2}+1\right)}$.

Finally, using (3.3) in the time-time component of the Einstein equations for the second metric we get that the ratio $\xi$ of the two scale parameters satisfies the following algebraic equation:

$$
\begin{aligned}
& \xi^{2}\left(\frac{8 a_{4}}{\kappa}-2 a_{2}\right)+\xi\left(\frac{6 a_{3}}{\kappa}-a_{1}\right)+\frac{a_{1}}{3 \kappa \xi}+\frac{2 a_{2}}{\kappa}-2 a_{3} \xi^{3}-\frac{a_{0}}{3} \\
& \quad=\frac{8 \pi G}{3 m^{2}}\left(\rho_{1}-\frac{\xi^{2} \rho_{2}}{\kappa}\right) .
\end{aligned}
$$

The analysis is identical when the same spatial curvature $k_{c}$ is introduced in (3.1) for both metrics. ${ }^{3}$ The presence of the second matter opens the possibility for a behavior of $\xi$ different from the one found in [23].

We assume that the mass scale $m$ is related to the present cosmological constant as $m^{2} M_{\mathrm{pl}}^{2} \propto \Lambda$ and the equation of state for matter one and two is such that $w_{1,2}>-1$. The assumption on the scale $m$ is natural if massive gravity is relevant for the present acceleration of the Universe. ${ }^{4}$ In order to not spoil early cosmology (say before nucleosynthesis until after the decoupling time), the contribution proportional to $m$ in (3.4) has to kick in only at small redshift $(z \sim 10)$ when "dark energy" starts to dominate the expansion rate. This is the case when

$$
\frac{3 \mathcal{H}^{2}}{a^{2}} \simeq 8 \pi G \rho_{1} \quad \text { implying } m^{2} \sum_{i=0}^{3}\left(a_{i} \xi^{i}\right) \ll 8 \pi G \rho_{1},
$$

or equivalently

$$
\frac{\Lambda}{\rho_{1}} \sum_{i=0}^{3} a_{i} \xi^{i} \ll 1
$$

Now, for most of the history of our Universe (matter and radiation periods) $\rho_{1} \gg \Lambda$, thus (3.10) is naturally satisfied unless $\xi$ evolves to values of $\sim \rho_{1} / \Lambda$. As a result, in such a regime, the implementation of Eq. (3.10) in Eq. (3.8) requires that at the leading order

$$
8 \pi G\left(\rho_{1} \kappa-\rho_{2} \xi^{2}\right) \simeq \begin{cases}\frac{a_{1} m^{2}}{\xi^{2}} & \text { when } \xi \ll 1 \\ 0 & \text { when } \xi \sim 1\end{cases}
$$

\footnotetext{
${ }^{3}$ The spatial curvatures must be equal for consistency [23].

${ }^{4} \mathrm{We}$ do not consider here the case [34] $\mathrm{m}^{2} \mathrm{M}_{\mathrm{pl}}^{2} \gg \rho_{1}$, where the scale of $m$ is not related with the present acceleration of the Universe.
}

In absence of a second matter sector, the solution $\xi \sim 1$ could not exist. Of course, when (3.9) holds, while the dynamics of $a$ is not affected by $\xi$, on the contrary, the impact on $\mathcal{H}_{\omega}$ can be relevant; see (3.2). According to Eq. (3.11), the following regimes for the background value of $\xi$ emerge:

(A) When $\xi^{2} \rho_{2} \gg \rho_{1} \gg \Lambda^{5}$

$$
\begin{aligned}
\xi & \simeq-\left(\frac{a_{1} m^{2}}{8 \pi G \rho_{2}}\right)^{1 / 3} \propto \frac{\Lambda^{1 / 3}}{\rho_{2}^{1 / 3}} \ll \frac{\Lambda}{\rho_{1}} \ll 1, \\
\text { with } \quad c & \simeq-\frac{1}{w_{2}} .
\end{aligned}
$$

The above expression can be rewritten also in the form

$$
\xi=\left(-\frac{8 \pi G \rho_{2}^{\mathrm{in}}}{a_{1} m^{2}}\right)^{\frac{1}{3 w_{2}}} a^{-\frac{1+w_{2}}{w_{2}}}
$$

where the explicit time dependence of $\xi$ is shown. The above expressions are valid when $w_{2}<0$. Clearly, we have that $c>0$ and we also need $a_{1}<0$ so that $\xi$ is real and positive. Being $\xi \ll 1$, (3.9) is satisfied. Requiring $w_{2}<0$ is rather exotic; nevertheless, as will be shown in Sec. IV B, it does not help to avoid instabilities.

(B) When $\rho_{1} \gg \xi^{2} \rho_{2}$ and at any time $\rho_{1} \gg \Lambda$.

This case was considered in [26] when a single matter sector was present. Clearly (3.9) is easily satisfied. The value for $\xi$ is of the form

$$
\xi \simeq \frac{a_{1} m^{2}}{8 \pi G \rho_{1} \kappa} \propto \frac{\Lambda}{\rho_{1}} \ll 1, \quad \text { with } \quad c \simeq\left(4+3 w_{1}\right),
$$

and self-consistency requires that

$$
\rho_{2} \ll \frac{\rho_{1}^{3}}{\Lambda^{2}}
$$

(C) When $\rho_{1} \simeq \xi^{2} \rho_{2} \gg \Lambda$

$$
\xi \simeq\left(\kappa \frac{\rho_{1}}{\rho_{2}}\right)^{1 / 2}=\xi_{\text {in }} a^{\frac{3\left(w_{1}-w_{2}\right)}{1+3 w_{2}}}, \quad \text { with } \quad c \simeq \frac{1+3 w_{1}}{1+3 w_{2}} ;
$$

where we used the solutions of Eq. (3.7) and $\xi_{\text {in }}=$ $\left(\kappa \rho_{1}^{\text {in }} / \rho_{2}^{\text {in }}\right)^{-1 /\left(1+3 w_{2}\right)}$ defines the initial time conditions in terms of the initial density ratio. In such a regime $\rho_{2} \propto a^{-3 \frac{\left(1+w_{2}\right)\left(1+3 w_{1}\right)}{1+3 w_{2}}}$, thus only when $w_{2}>-\frac{1}{3}$ matter density in the second sector decreases with time, while

\footnotetext{
${ }^{5}$ Notice that, being $\xi^{2} \gg \frac{\Lambda}{\rho_{2}} \simeq \xi^{3}$, then $\xi \ll 1$ and so we are in the region where $\rho_{2} \gg \rho_{1} \gg \rho_{2}$.
} 
$\xi$ can grow or decay depending on the sign of $\left(w_{1}-w_{2}\right)$. When $w_{2}>w_{1}$, going back in time, $\xi$ grows; nevertheless, condition (3.10) is still satisfied if $w_{1} \geq 0$. The validity region of such an approximated solution is in the range

$$
\frac{\Lambda}{\rho_{1}} \ll \xi \ll\left(\frac{\rho_{1}}{\Lambda}\right)^{n}
$$

where the power $n$ can be $1 / 3,1 / 2$, or 1 depending on the $a_{i}$ values; see [23] for details. When $w_{1}>w_{2}$ and $\xi$ decreases going back in time, the above lower bound holds for $w_{2}>-1 /\left(4+3 w_{1}\right)$.

(D) When $\rho_{2}=0$, also the case of very large $\xi$ is possible, with

$$
\xi \propto\left(\frac{\rho_{1}}{\Lambda}\right)^{n}
$$

which gives $c<0$. The power $n$ is the same as in Eq. (3.17); see [23]. Thus, not only is (3.9) violated but also $c$ is negative. Starting from a negative $c$ in order to get to a quasi-dS phase, where $c \sim 1$, one has to cross $c=0$ where $\tilde{g}$ is singular. ${ }^{6}$

Finally, looking at the validity of our approximation, we found that the explored range of the $\xi$ values can be divided in the following disjoined regions:

$$
\xi_{(\mathrm{A})} \ll \xi_{(\mathrm{B})} \sim \frac{\Lambda}{\rho_{1}} \ll \xi_{(\mathrm{C})} \ll \xi_{(\mathrm{D})} \sim\left(\frac{\rho_{1}}{\Lambda}\right)^{n}
$$

which cover the whole range of $\xi$; except (D), all cases are compatible with Eq. (3.9), i.e., an early time standard FRW universe.

\section{PERTURBED FRW UNIVERSE}

Perturbations around the solution (3.1) can be studied along the same lines of [26]. We focus here on the scalar sector; in the vector and tensor ones, the results are very similar to the case with only $\rho_{1}$ and they can be found in [26]. In the scalar sector we have eight fields and two independent gauge transformations; as a result we can form six independent gauge invariant scalar combinations $\Psi_{1}, \Psi_{2}, \Phi_{1}, \Phi_{2}, \mathcal{E}, \mathcal{B}_{1}$ for the metric perturbations. For matter we have the gauge invariant density perturbations $\delta \rho_{1 / 2 \mathrm{gi}}$ and the scalar part of velocity perturbations $\delta u_{s 1 / 2}$. The various definitions can be found in Appendix A where also the full set of equations is given.

The fields $\mathcal{B}_{1}$ and $\Psi_{1 / 2}$ are nondynamical and can be expressed in terms of $\mathcal{E}$ and $\Phi_{1 / 2}$, in particular,

\footnotetext{
${ }^{6}$ This point was overlooked in $[24,35,36]$. We only consider FRW-like backgrounds where $c>0$.
}

$$
\Psi_{1}+\Phi_{1}=m^{2} a^{2} f_{1} \mathcal{E}, \quad \Psi_{2}+\Phi_{2}=-\frac{m^{2} a^{2} f_{1} \mathcal{E}}{\kappa c \xi^{2}} ;
$$

where $f_{1 / 2}$ are defined in Eq. (A13). The fields $\mathcal{E}$ and $\Phi_{1 / 2}$ satisfy three second-order equations; thus 3 scalar DoF propagate.

The condition (3.9) guarantees only that the background solution follows closely GR cosmology with standard matter (sector 1) until the present epoch. Of course we need more than that: we need to be sure that perturbations, in particular the ones related to the new degrees of freedom, do not start growing too early. Indeed, that is precisely what happens when only ordinary matter is present: at early time, the mode $\Phi_{2}$ inside the horizon grows exponentially, though $\Phi_{1}$ and $\delta=\delta \rho_{1 \mathrm{gi}} / \rho_{1}$ are the same as in GR. As a result we have to face a very early breakdown of perturbation theory. Apparently, this point was not taken into account fitting the parameters $a_{i}$ and $m$ against observations [37]. Basically, in the presence of the aforementioned instabilities, structure formation will be completely different. Thus, a preliminary necessary condition is to get rid of exponential instabilities, irrespective of their tachyonic or ghost nature. In what follows we will show that also the presence of a second matter sector is not instrumental in avoiding such kinds of instabilities.

\section{A. Structure of the evolution equations}

The equations are rather complicated; however, at early times we can expand using the small parameter $\epsilon=$ $m \mathcal{H}^{-1} \sim\left(\Lambda / \rho_{1}\right)^{1 / 2}$. Formally this is equivalent to expanding the equations of the perturbation for small $m$. We stress that in the $m \rightarrow 0$ limit there is no guarantee to recover GR as discussed in detail in [26].

In all cases (A), (B), and (C), $\Phi_{1}$, at leading order in $\epsilon$, satisfies the following equation:

$$
\Phi_{1}^{\prime \prime}+\frac{6\left(w_{1}+1\right)}{\left(1+3 w_{1}\right) \tau} \Phi_{1}^{\prime}+k^{2} w_{1} \Phi_{1}=0,
$$

which coincides with the one in GR. In the radiation epoch, subhorizon modes oscillate, dumped by a factor $a^{2}$, while superhorizon modes are frozen and $\Phi_{1}=$ constant. In a matter dominated Universe $\Phi_{1}$ is always constant. Thus, at leading order in $\epsilon$, the dynamics of $\Phi_{2}$ and $\mathcal{E}$ is described by a system of coupled second-order ordinary differential equations of the form

$$
\begin{aligned}
\phi^{\prime \prime}+\mathcal{D} \phi^{\prime}+\mathcal{M} \phi+z_{1} \Phi_{1}+z_{2} \Phi_{1}^{\prime} & =0 \\
\phi & =\left(\begin{array}{c}
\Phi_{2} \\
\mathcal{E}_{N} \equiv \mathcal{E} / \tau^{2}
\end{array}\right),
\end{aligned}
$$

where $\mathcal{D}$ and $\mathcal{M}$ are suitable $2 \times 2$ matrices and $z_{1 / 2}$ functions of $\tau$ and $k$. We have also conveniently introduced 
a dimensionless field $\mathcal{E}_{N}=\mathcal{E} / \tau^{2}$. Thus, once $\Phi_{1}$ is found from (4.2), it enters in (4.3) as a source term. As shown in Appendix B, Eqs. (4.3) correspond, for subhorizon and superhorizon modes, to a coupled system of Bessel-like equations. It turns out that for cases (A) and (C), the system (4.3) further simplifies because the dynamics of $\Phi_{2}$ decouples from the one of $\mathcal{E}_{N}$ and stability can be established simply by studying the mass term. For the case (B), on the contrary, one has to do a more involved analysis.

\section{B. Case (A)}

One has to be careful in the expansion; indeed here one can expand for small $m$ only if $w_{2}<-1 / 3$. The result, this time, is that also the equation for $\Phi_{2}$ is decoupled. In particular, we have that

$$
\begin{aligned}
& \Phi_{2}^{\prime \prime}+\frac{6\left(1-\left|w_{2}\right|\right)}{\left|w_{2}\right|\left(3 w_{1}+1\right) \tau} \Phi_{2}^{\prime}-\left(\frac{k^{2}}{\left|w_{2}\right|}+\frac{\left|w_{2}\right|\left(3 w_{1}+4\right)-4}{w_{2}^{2} \tau^{2}\left(3 w_{1}+1\right)^{2}}\right) \\
& \Phi_{2}=0 .
\end{aligned}
$$

The above equation can be easily solved in terms of Bessel functions. However, it is clear that the solution has an exponentially growing mode. Indeed, inside the horizon $x=k \tau \gg 1$, the mass term is simply proportional to $-\left|w_{2}\right|$ and is negative. The solution reads

$$
\begin{aligned}
\Phi_{2} & =\left(x\left|w_{2}\right|\right)^{2-\frac{3}{2\left|w_{2}\right|}}\left[\alpha_{1} J_{\nu}\left(\frac{-i x}{\sqrt{\left|w_{2}\right|}}\right)+\alpha_{2} Y_{\nu}\left(\frac{-i x}{\sqrt{\left|w_{2}\right|}}\right)\right], \\
\nu & =\frac{\sqrt{4 w_{2}\left(4 w_{2}+1\right)+5}}{2\left|w_{2}\right|} .
\end{aligned}
$$

Clearly $\Phi_{2}$ grows like $e^{x / \sqrt{\left|w_{2}\right|}}$. The same instability is present also for the field $\mathcal{E}$ whose mass term, inside the horizon, gets the value $\frac{k^{2}\left(w_{1}-1\right)}{\left|w_{2}\right|\left(3 w_{1}+1\right)}$. As a result, exponential instabilities are always present in both $\Phi_{2}$ and $\mathcal{E}$ and the background (A) is pathological. The behavior of superhorizon modes is similar to the case (B), discussed bellow.

\section{Case (B)}

As for the case (A), the expansion for small $m$ is a bit tricky, indeed $\xi \simeq \frac{a_{1} m^{2}}{8 \pi G \rho_{1} \kappa}$ goes to zero when $m \rightarrow 0$ and all quantities must be expanded to next-to-leading order. In this case, as shown in Appendix B, the equations for $\Phi_{2}$ and $\mathcal{E}$ stay coupled. The only way to decouple them is to work with a fourth-order equation for one of the two fields. Taking for simplicity $w_{1}=1 / 3$, we get for subhorizon modes

$$
\begin{gathered}
\mathcal{E}_{N}^{(4)}+\frac{5\left(3 w_{2}+5\right)}{\tau} \mathcal{E}_{N}^{(3)}+k^{2}\left(25 w_{2}-\frac{5}{3}\right) \mathcal{E}_{N}^{\prime \prime} \\
+k^{2} \frac{25\left(9 w_{2}-1\right)}{\tau} \mathcal{E}_{N}^{\prime}-k^{4} \frac{125 w_{2}}{3} \mathcal{E}_{N}=0
\end{gathered}
$$

and for $w_{1}=0$

$$
\begin{aligned}
\mathcal{E}_{N}^{(4)} & +\frac{8\left(3 w_{2}+5\right) \mathcal{E}_{N}^{(3)}}{\tau}+k^{2}\left(16 w_{2}-1\right) \mathcal{E}_{N}^{(2)} \\
& +\frac{8 k^{2}\left(29 w_{2}-3\right)}{\tau} \mathcal{E}_{N}^{\prime}-k^{4} 16 w_{2} \mathcal{E}+\frac{k^{2}\left(20 w_{2}+3\right)}{\tau} \Phi_{1}^{\prime} \\
& +\frac{8 k^{4} w 2}{3} \Phi_{1}=0 .
\end{aligned}
$$

Even before attempting solving (4.6) and (4.7) one sees that an exponential instability is expected. Indeed, for $\mathcal{D}$ and $\mathcal{M}$ in (4.3) we have that

$$
\begin{aligned}
\operatorname{Det}(\mathcal{D}) & =\frac{24\left(3 w_{1}+4\right)^{2}\left(w_{2}+1\right)}{\tau^{2}\left(3 w_{1}+1\right)^{2}}, \\
\operatorname{Tr}(\mathcal{D}) & =\frac{2\left(4+3 w_{1}\right)\left(5+3 w_{2}\right)}{\tau\left(1+3 w_{1}\right)}, \\
\operatorname{Det}(\mathcal{M}) & =-k^{4}\left(2 w_{1}+1\right)\left(3 w_{1}+4\right)^{2} w_{2}, \\
\operatorname{Tr}(\mathcal{M}) & =k^{2}\left[\left(4+3 w_{1}\right)^{2} w_{2}-2 w_{1}-1\right] .
\end{aligned}
$$

Thus, while $\mathcal{D}$ is positive definite, $\mathcal{M}$ has at least a negative eigenvalue; in particular, the eigenvalues of $\mathcal{M}$ are given by

$$
\lambda_{1}=-k^{2}\left(2 w_{1}+1\right), \quad \lambda_{2}=k^{2}\left(3 w_{1}+4\right)^{2} w_{2} .
$$

Clearly, the fact that $\lambda_{1}<0$ will lead to an exponential growth of subhorizon modes. It should be stressed that $\lambda_{1}$ does not depend on $w_{2}$ and precisely coincides with the negative mass term of $\mathcal{E}_{N}$ found in the case where a single matter sector was present [26]. ${ }^{7}$ The numerical solution of (4.6) and (4.7) confirms that there is no value of $w_{2}$ such that $\mathcal{E}_{N}$ does not grow exponentially. It is evident that subhorizon instability cannot be avoided.

For superhorizon modes we can give directly the full solutions

$$
\begin{aligned}
\mathcal{E}= & \overline{\mathcal{E}}_{1} \tau^{-15 w_{2}-1}+\overline{\mathcal{E}}_{2} \tau^{-\frac{9}{2}-\frac{\sqrt{21}}{2}}+\overline{\mathcal{E}}_{3} \tau^{\frac{1}{2}(\sqrt{21}-9)}+\frac{\overline{\mathcal{E}}_{4}}{\tau^{7}} \\
& -\frac{32 \tau^{2} \bar{\Phi}_{1}}{37} \text { for } w_{1}=\frac{1}{3}, \\
\mathcal{E}= & \overline{\mathcal{E}}_{1} \tau^{-24 w_{2}-4}+\overline{\mathcal{E}}_{2} \tau^{-\frac{15}{2}-\frac{\sqrt{33}}{2}}+\overline{\mathcal{E}}_{3} \tau^{\frac{1}{2}(\sqrt{33}-15)} \\
& +\frac{\overline{\mathcal{E}}_{4}}{\tau^{13}}-\frac{21 \tau^{2} \bar{\Phi}_{1}}{82} \text { for } w_{1}=0,
\end{aligned}
$$

where $\overline{\mathcal{E}}_{i}$ are the values of $\mathcal{E}$ at same initial time and $\bar{\Phi}_{1}$ is the frozen value of $\Phi_{1}$. Notice that in particular for $w_{1}=\frac{1}{3}$ the nondecaying mode of the three perturbations are

\footnotetext{
${ }^{7}$ For reference, when $\rho_{2}=0, \Phi_{2}$ has a tachyonic mass equal to $\lambda_{1}$ and $\mathcal{E}_{N}=-\frac{2}{3} \Phi_{2}$, for $w_{1}=1 / 3$.
} 


$$
\Phi_{1}=\bar{\Phi}_{1} \quad \Phi_{2}=\frac{39}{37} \bar{\Phi}_{1}, \quad \mathcal{E}=-\frac{32}{37} \tau^{2} \bar{\Phi}_{1} .
$$

In the metric perturbations actually $\mathcal{E}$ enters with the combination $k^{2} \mathcal{E} \propto(k \tau)^{2} \ll 1$, and as a result it stays very small and there are no consequences on the validity of the perturbative expansion. In addition, from (4.1) we get that

$$
\Psi_{1}+\Phi_{1} \approx 0, \quad \Psi_{2}+\Phi_{2} \approx \frac{96}{185} \bar{\Phi}_{1} .
$$

Thus, perturbations in the sector one, relevant for our matter, are indistinguishable from GR at early times. In the second sector the two Bardeen potentials are not equal even if the source is a perfect fluid.

\section{Case (C)}

As shown in Appendix B, also in this case the dynamics of $\Phi_{2}$ is decoupled and its equations are similar to the ones in case (A). Inside the horizon, simply looking at the timedependent mass terms we find that they are positive, avoiding instabilities, when (see Appendix B)

$$
w_{1 / 2}>0, \quad 3 w_{1}+1-\frac{4 f_{1}}{f_{2}}>0,
$$

where $f_{1,2}$, see Eq. (A13), are $\tau$ dependent. Notice that when $f_{1}=f_{2}$ the above condition cannot be satisfied if $0 \leq w_{1}<1$. Actually, we have $f_{1}=f_{2}$ when $c=1$ and/or $a_{2}=a_{3}=0$ (as in the simplest bigravity model of [37]), and also when $w_{1}=w_{2}$. Now depending on whether $w_{1}>$ $w_{2}$ or $w_{2}>w_{1}, \xi$ dynamically becomes very small or very large in the early universe, being

$$
\xi(\tau)=\xi_{\text {in }} a^{\frac{3\left(w_{1}-w_{2}\right)}{\left(1+3 w_{2}\right)}} .
$$

In particular

$$
3 w_{1}+1-\frac{4 f_{1}}{f_{2}}=\left\{\begin{array}{ll}
\frac{3\left(3 w_{1}+1\right)\left(w_{2}-1\right)}{3 w_{2}+1}<0 & \text { for } \xi \rightarrow \infty \\
3\left(w_{1}-1\right)<0 & \text { for } \xi \rightarrow 0
\end{array} .\right.
$$

Thus, (4.14) cannot be satisfied at early times. When $\xi \rightarrow 0$ or $\xi \rightarrow \infty$, the mass term of $\mathcal{E}$ becomes time independent and negative definite, leading to an exponential instability. We conclude therefore that also in the case (C) the instability cannot be avoided if $w_{2}<1$. For what concerns superhorizon modes the discussion is similar to the case (B).

\section{CONCLUSIONS}

We studied in detail the dynamics of scalar perturbations in massive bigravity. Beside its theoretical interest, massive gravity could be an interesting alternative to dark energy. As a general ground, the ghost free massive gravity theories can be classified according to the global symmetries of the potential $V$ in the unitary gauge [18]. The ones characterized by Lorentz invariance on flat space have a number of issues once an homogeneous FRW background is implemented.

In the bigravity formulation, with a single matter sector, things get better and FRW cosmological solutions indeed exist [23-25]. However, cosmological perturbations are different from the ones in GR. Already during radiation domination, subhorizon scalar perturbations tend to grow exponentially [26]. The manifestation of such instabilities is rather peculiar. In the sector one, composed by ordinary matter and the metric $g$, their perturbations are very close to the ones of GR. The instability manifests as an exponential subhorizon growth of the field $\mathcal{E}$ and of the second scalar mode $\Phi_{2}$, one of the Bardeen potentials of $\tilde{g}$, which quickly invalidates the use of perturbation theory at very early time. This is very different from GR where perturbations become large (power law growth) only when the Universe is nonrelativistic.

The emergence of an instability only in the perturbations of the second metric suggests its origin may reside in the matter content asymmetry of the two sectors, since only the physical metric is coupled to matter. Indeed, the only background solutions acceptable have a ratio $\xi=\omega / a$ of the metrics' scale factors such that $\xi \ll 1$.

Adding a second matter sector sourcing the second metric opens up the possibility [case $(\mathrm{C})$ ] to have a more symmetric background with $\xi \sim 1$ and one may hope the exponential instability is absent. Unfortunately, we have shown that this is not the case. Though, the pressure provided by the second matter stabilizes $\Phi_{2}$ and its dynamics becomes similar to GR, the subhorizon instability persists for $\mathcal{E}$ that represents a purely gravitational extra scalar field.

We managed to analyze the perturbations in the whole range of $\xi$ compatible with the early Universe evolution (matter and radiation). The cases (A) and (B) represent regions of very small $\xi$ where only one matter sector dominates; likewise the case with a single matter, and both $\mathcal{E}$ and $\Phi_{2}$ grow exponentially inside the horizon. When $\rho_{1} \gg \rho_{2}$, the values of the tachyonic mass responsible for that instability does not depend on $w_{2}$ and actually coincides with the one found in the case where $\rho_{2}=0$ [26]. In region (C) both the matter sectors are important. While the Bardeen potentials $\Phi_{1,2}$ are stable, the purely scalar gravitational field $\mathcal{E}=E_{1}-E_{2}$ (see Appendix A) that involves both metrics has early time instabilities. Finally, the region (D), characterized by very large values of $\xi$, already at the level of background, spoils early time standard FRW cosmology.

Spanning the whole range of $\xi$ compatible with a standard early time cosmology, when $m^{2} M_{\mathrm{pl}}^{2}$ is the order of the present cosmological constant, the bottom line is that massive bigravity has an intrinsic exponential instability.

Looking at the behavior of the matter contrast that is the same of GR, one may speculate that some sort of Vainshtein [38] cosmological mechanism could take place, though 
FRW COSMOLOGICAL PERTURBATIONS IN MASSIVE ...

here the trouble is with perturbations and not with the background. Even if that happens, the deal is rather pricey: perturbation theory will fail both at Solar System and cosmological scales.

\section{ACKNOWLEDGEMENTS}

M. C. thanks A. Emir Gümrükçüoğlu for useful discussions and the Fondazione Angelo Della Riccia for financial support. L.P. thanks the Cosmology and Astroparticle Physics Group of the University of Geneva for hospitality and support. D. C. thanks Negramaro for their Senza fiato inspiring song.

Note added.-After the completion of this paper, [39] came out confirming our results. In addition, [39] shows that cosmological perturbations around the background solution found in [34] with $m^{2} \gg H_{0}^{2}$ (i.e., the mass $m$ is not related with the current Hubble scale $H_{0}$ ) are stable. However, the coefficients $a_{i}$ of the potential must be subject to a huge tuning to reproduce the present acceleration of the Universe.

\section{APPENDIX A: PERTURBED GEOMETRY}

Let us now consider the perturbations of the FRW background (3.1)

$$
g_{\mu \nu}=\bar{g}_{1 \mu \nu}+a^{2} h_{1 \mu \nu}, \quad \tilde{g}_{\mu \nu}=\bar{g}_{2 \mu \nu}+\omega^{2} h_{2 \mu \nu},
$$

parametrized as follows:

$$
\begin{aligned}
h_{100} \equiv & -2 A_{1}, \quad h_{200} \equiv-2 c^{2} A_{2}, \\
h_{1 / 20 i} \equiv & \mathcal{C}_{1 / 2 i}-\partial_{i} B_{1 / 2}, \\
\partial^{i} \mathcal{V}_{1 / 2 i}= & \partial^{i} \mathcal{C}_{1 / 2 i}=\partial^{j} h^{T T}{ }_{1 / 2 i j}=\delta^{i j} h^{T T}{ }_{1 / 2 i j}=0, \\
h_{1 / 2 i j} \equiv & h^{T T}{ }_{1 / 2 i j}+\partial_{i} \mathcal{V}_{1 / 2 j}+\partial_{j} \mathcal{V}_{1 / 2 i}+2 \partial_{i} \partial_{j} E_{1 / 2} \\
& +2 \delta_{i j} F_{1 / 2} .
\end{aligned}
$$

Spatial indices are raised or lowered using the spatial flat metric. In the scalar sector we can form six independent gauge invariant scalar combinations that we chose to be

$$
\begin{aligned}
\Psi_{1} & =A_{1}-\mathcal{H} \Xi_{1}-\Xi_{1}^{\prime} \\
\Psi_{2} & =A_{2}+c^{-2}\left(\frac{c^{\prime}}{c}-\mathcal{H}_{\omega}\right) \Xi_{2}-\frac{\Xi_{2}^{\prime}}{c^{2}} \\
\Phi_{1} & =F_{1}-\mathcal{H} \Xi_{1}, \quad \Phi_{2}=F_{2}-\mathcal{H}_{\omega} \frac{\Xi_{2}}{c^{2}}, \\
\mathcal{E} & =E_{1}-E_{2}, \quad \mathcal{B}_{1}=B_{2}-c^{2} B_{1}+\left(1-c^{2}\right) E_{1}^{\prime},
\end{aligned}
$$

where $\Xi_{1 / 2}=B_{1 / 2}+E_{1 / 2}^{\prime}$. In the matter sectors, we define the following gauge invariant perturbed pressure and density:
PHYSICAL REVIEW D 90, 084003 (2014)

$$
\begin{aligned}
& \delta \rho_{1_{g i}}=\delta \rho_{1}-\Xi_{1} \rho_{1}^{\prime}, \quad \delta p_{1_{g i}}=\delta p_{1}-\Xi_{1} p_{1}^{\prime} ; \\
& \delta \rho_{2_{g i}}=\delta \rho_{2}-\frac{\Xi_{2}}{c^{2}} \rho_{2}^{\prime}, \quad \delta p_{2 i}=\delta p_{2}-\frac{\Xi_{2}}{c^{2}} p_{2}^{\prime} .
\end{aligned}
$$

The scalar part $v$ of the perturbed 4-velocity $u^{\mu}$ is defined as

$$
\begin{aligned}
u_{1 / 2}^{\mu} & =\bar{u}_{1 / 2}^{\mu}+\delta u^{\mu}, \quad u_{1}^{\mu} u_{1}^{\nu} g_{\mu \nu}=-1, \\
u_{2}^{\mu} u_{2}^{\nu} \tilde{g}_{\mu \nu} & =-1, \quad \delta u_{1 / 2}^{0}=-a^{-1} A_{1 / 2} ; \\
\delta u_{1 / 2 i} & =a\left(\partial_{i} v_{1 / 2}-\partial_{i} B_{1 / 2}\right) .
\end{aligned}
$$

The corresponding gauge invariant quantity is defined as

$$
u_{1 / 2 s}=v+E_{1 / 2}^{\prime} .
$$

The conservation of the energy momentum tensor leads to a set of differential relations. For the sector 1 we have

$$
\begin{aligned}
\delta \rho_{1_{g i}}^{\prime} & =\left(1+w_{1}\right)\left[\rho_{1}\left(k^{2} u_{1 s}-3 \Phi_{1}^{\prime}\right)-3 \mathcal{H} \delta \rho_{1_{g i}}\right] \\
u_{1 s}^{\prime} & =\left(3 w_{1}-1\right) u_{1 s} \mathcal{H}-\frac{w_{1}}{\left(1+w_{1}\right)} \frac{\delta \rho_{1_{g i}}}{\rho_{1}}-\Psi_{1} .
\end{aligned}
$$

For the sector 2,

$$
\begin{aligned}
\delta \rho_{2_{g i}}^{\prime} & =\left(1+w_{2}\right)\left[\rho_{2}\left(k^{2} u_{2 s}-3 \Phi_{2}^{\prime}\right)-3 \mathcal{H}_{\omega} \delta \rho_{2_{g i}}\right] \\
u_{2 s}^{\prime} & =u_{2 s}\left[\left(3 w_{2}-1\right) \mathcal{H}_{\omega}+\frac{c^{\prime}}{c}\right]-c^{2}\left[\frac{w_{2}}{\left(1+w_{2}\right)} \frac{\delta \rho_{2_{g i}}}{\rho_{2}}+\Psi_{2}\right] .
\end{aligned}
$$

The perturbed Einstein equations for the first metric reads

$$
\begin{aligned}
& 2 \Delta \Phi_{1}+6 \mathcal{H}\left(\Psi_{1} \mathcal{H}-\Phi_{1}^{\prime}\right)+a^{2} m^{2} f_{2}\left(3 \mathcal{F}_{1}-\Delta \mathcal{E}\right) \\
& \quad=-8 \pi a^{2} G \delta \rho_{1_{g i}} ; \\
& \partial_{i}\left[2 \Psi_{1} \mathcal{H}-2 \Phi_{1}^{\prime}+\frac{a^{2} m^{2} \mathcal{B}_{1} f_{2}}{(c+1)}+8 \pi G a^{2}\left(p_{1}+\rho_{1}\right) u_{1 s}\right]=0
\end{aligned}
$$

$$
\begin{aligned}
& \left(\partial_{i} \partial_{j}-\delta_{i j} \Delta\right)\left(a^{2} f_{1} m^{2} \mathcal{E}-\Phi_{1}-\Psi_{1}\right) \\
& \quad+\delta_{i j}\left[m^{2} a^{2}\left(2 f_{1} \mathcal{F}_{1}+f_{2} \mathcal{A}_{1}\right)+2 \Psi_{1}\left(\mathcal{H}^{2}+2 \mathcal{H}^{\prime}\right)\right. \\
& \left.-2 \Phi_{1}^{\prime \prime}-2 \mathcal{H}\left(2 \Phi_{1}^{\prime}-\Psi_{1}^{\prime}\right)\right]=8 \pi G a^{2} \delta_{i j} \delta p_{1_{g i}}
\end{aligned}
$$

where

$$
\begin{aligned}
& f_{1}=\xi\left[2 \xi\left(3 a_{3} c \xi+a_{2}(c+1)\right)+a_{1}\right], \\
& f_{2}=\xi\left(6 a_{3} \xi^{2}+4 a_{2} \xi+a_{1}\right) .
\end{aligned}
$$

For the metric $\tilde{g}$ 


$$
\begin{aligned}
& 2 c^{2} \Delta \Phi_{2}+6 \mathcal{H}_{\omega}\left(\Psi_{2} \mathcal{H}_{\omega}-\Phi_{2}^{\prime}\right)+\frac{m^{2} a^{2} f_{2}}{\kappa \xi^{2}} c^{2}\left(\Delta \mathcal{E}-3 \mathcal{F}_{2}\right) \\
& =-\frac{8 \pi G}{\kappa} a^{2} c^{2} \xi^{2} \delta \rho_{2_{g i}} \\
& \partial_{i}\left[2 c\left(\Psi_{2} \mathcal{H}_{\omega}-\Phi_{2}^{\prime}\right)-\frac{m^{2} a^{2} f_{2}}{\kappa \xi^{2}(1+c)} \mathcal{B}_{2}\right. \\
& \left.\quad+\frac{8 \pi G}{\kappa} c a^{2} \xi^{2}\left(p_{2}+\rho_{2}\right) u_{2 s}\right]=0 \\
& +c\left(\partial_{i} \partial_{j}-\delta_{i j} \Delta\right)\left[\frac{a^{2} f_{1} m^{2}}{\kappa \xi^{2}} \mathcal{E}+c\left(\Phi_{2}+\Psi_{2}\right)\right] \\
& +\delta_{i j}\left[\frac{m^{2} a^{2}}{\kappa \xi^{2}}\left(2 c f_{1} \mathcal{F}_{2}+f_{2} \mathcal{A}_{2}\right)\right. \\
& +2\left(\mathcal{H}_{\omega}^{2}+2 \mathcal{H}_{\omega}^{\prime}-2 \frac{c^{\prime}}{c} \mathcal{H}_{\omega}\right) \\
& \left.\times \Psi_{2}-2 \Phi_{2}^{\prime \prime}+2\left(\frac{c^{\prime}}{c}-2 \mathcal{H}_{\omega}\right) \Phi_{2}^{\prime}+2 \mathcal{H}_{\omega} \Psi_{2}^{\prime}\right] \\
& =\frac{8 \pi G}{\kappa} a^{2} c^{2} \xi^{2} \delta_{i j} \delta p_{2_{g i}}
\end{aligned}
$$

The gauge invariant fields $\mathcal{F}_{1 / 2}$ can be expressed in terms of $\Phi_{1 / 2}, \mathcal{B}_{1}$ and $\mathcal{E}$ by using

$$
\begin{gathered}
\mathcal{H}_{\omega} \mathcal{F}_{2}-\mathcal{H} \mathcal{F}_{1}=\left(\mathcal{H}-\mathcal{H}_{\omega}\right)\left(\Phi_{1}-\Phi_{2}\right) ; \\
c^{2}\left(\mathcal{F}_{2}+\mathcal{F}_{1}\right)=\left(\mathcal{B}_{1}-\mathcal{E}\right)\left(\mathcal{H}+\mathcal{H}_{\omega}\right)-2 c^{2}\left(\Phi_{1}-\Phi_{2}\right) .
\end{gathered}
$$

We often use the Fourier transform of perturbations with respect to $x^{i}$, the corresponding 3-momentum is $k^{i}$ and $k^{2}=k^{i} k_{i}$. To keep notation as simple as possible we give up the symbol of the Fourier transform.

\section{APPENDIX B: EVOLUTION OF PERTURBATIONS}

In this Appendix we give the equations that govern the evolution of the perturbations. We are interested in two regimes: subhorizon modes with $k \tau \gg 1$ and superhorizon ones for which $k \tau \ll 1$.

\section{Case (B)}

Remember that in this case we have $\xi \simeq \frac{a_{1} m^{2}}{8 \pi G \rho_{1} \kappa} \ll 1$ and that the leading contribution for the evolution equation of $\Phi_{1}$ is the same as GR. For subhorizon modes, $\Phi_{2}$ and $\mathcal{E}$ satisfy a system of coupled equations,

$$
\begin{aligned}
\Phi_{2}^{\prime \prime} & +\frac{6\left[9\left(w_{2}+1\right) w_{1}^{2}+3\left(5 w_{2}+7\right) w_{1}+4 w_{2}+14\right]}{\tau\left(3 w_{1}+4\right)\left(3 w_{1}+1\right)} \Phi_{2}^{\prime}+k^{2}\left[\left(3 w_{1}+1\right)\left(3 w_{1}+4\right) w_{2}-3 w_{1}-2\right] \Phi_{2} \\
& +k^{2}\left(3 w_{1}-1\right) \Phi_{1}+\frac{18 w_{1}-6}{\tau\left(3 w_{1}+1\right)} \Phi_{1}^{\prime}+\frac{12\left[9\left(3 w_{2}+1\right) w_{1}^{2}+15 w_{1}\left(3 w_{2}+1\right)+2\left(6 w_{2}+5\right)\right]}{\tau^{3}\left(3 w_{1}+1\right)^{3}\left(3 w_{1}+4\right)} \mathcal{E}^{\prime} \\
& +k^{2} \frac{6\left(3 w_{1}+1\right)\left(3 w_{1}+4\right) w_{2}-6\left(w_{1}+1\right)}{\tau^{2}\left(3 w_{1}+1\right)^{2}} \mathcal{E}=0 ; \mathcal{E}^{\prime \prime}+\frac{6\left(9 w_{2} w_{1}+9 w_{1}+12 w_{2}+10\right)}{\tau\left(9 w_{1}^{2}+15 w_{1}+4\right)} \mathcal{E}^{\prime}+k^{2}\left[w_{1}+3\left(3 w_{1}+4\right) w_{2}+1\right] \mathcal{E} \\
& -\frac{1}{6} k^{2} \tau^{2}\left(3 w_{1}-1\right)\left(3 w_{1}+1\right)^{2} \Phi_{1}+\tau\left(1-9 w_{1}^{2}\right) \Phi_{1}^{\prime}+\frac{1}{6} k^{2} \tau^{2}\left(3 w_{1}+1\right)^{2}\left(12 w_{2}+w_{1}\left(9 w_{2}+3\right)+2\right) \Phi_{2} \\
& +\frac{\tau\left(3 w_{1}+1\right)\left[12 w_{2}+w_{1}\left(9 w_{2}+3\right)+2\right]}{3 w_{1}+4} \Phi_{2}^{\prime}=0 .
\end{aligned}
$$

For superhorizon modes, the coupled equations for $\Phi_{2}$ and $\mathcal{E}$ are given by

$$
\begin{gathered}
\Phi_{2}^{\prime \prime}+\frac{6\left(3 w_{1}+4\right)\left(w_{2}+1\right)}{\tau\left(3 w_{1}+1\right)} \Phi_{2}^{\prime}+\frac{6\left(15 w_{1}+17\right)\left[\left(3 w_{1}+4\right) w_{2}+1\right]}{\tau^{2}\left(3 w_{1}+1\right)^{2}} \Phi_{2}+\frac{24}{\tau^{3}\left(3 w_{1}+1\right)^{3}} \mathcal{E}^{\prime}+\frac{48\left[12 w_{2}+w_{1}\left(9 w_{2}-3\right)+2\right]}{\tau^{4}\left(3 w_{1}+1\right)^{4}} \mathcal{E} \\
-\frac{18\left(3 w_{1}+5\right)\left(\left(3 w_{1}+4\right) w_{2}+1\right)}{\tau^{2}\left(3 w_{1}+1\right)^{2}} \Phi_{1}-\frac{18\left[\left(3 w_{1}+4\right) w_{2}+1\right]}{\tau\left(3 w_{1}+1\right)} \Phi_{1}^{\prime}=0, \\
\mathcal{E}^{\prime \prime}+\frac{2\left(3 w_{1}+7\right)}{\tau\left(3 w_{1}+1\right)} \mathcal{E}^{\prime}+\frac{36\left(3 w_{1}^{2}+\left(6 w_{2}+5\right) w_{1}+8 w_{2}+2\right)}{\tau^{2}\left(3 w_{1}+1\right)^{3}} \mathcal{E}-\tau\left(36 w_{2}+3 w_{1}\left(9 w_{2}-2\right)+1\right) \Phi_{1}^{\prime} \\
-\frac{9\left(3 w_{1}+5\right)\left(4 w_{2}+w_{1}\left(3 w_{2}-1\right)\right)}{3 w_{1}+1} \Phi_{1}+\tau\left(3\left(3 w_{1}+4\right) w_{2}+1\right) \Phi_{2}^{\prime} \\
+\frac{3\left(9 w_{1}^{3}+9\left(5 w_{2}+2\right) w_{1}^{2}+\left(111 w_{2}+14\right) w_{1}+68 w_{2}+7\right)}{3 w_{1}+1} \Phi_{2}=0 .
\end{gathered}
$$




\section{Case (C)}

For this case $\xi \simeq\left(\frac{\kappa \rho_{1}}{\rho_{2}}\right)^{1 / 2}=\xi_{\text {in }} a^{\frac{3\left(w_{1}-w_{2}\right)}{1+3 w_{2}}}$. At the leading order in the $\epsilon$ expansion, $\Phi_{2}$ satisfies the following equation that is valid for any $k \tau$

$$
\Phi_{2}^{\prime \prime}+\frac{6\left(w_{2}+1\right)}{\tau\left(3 w_{2}+1\right)} \Phi_{2}^{\prime}+k^{2} \frac{w_{2}\left(3 w_{1}+1\right)^{2}}{\left(3 w_{2}+1\right)^{2}} \Phi_{2}=0
$$

For $\mathcal{E}$, inside the horizon, we get

$$
\begin{aligned}
\mathcal{E}^{\prime \prime} & +\frac{2\left[2 f_{1}\left(3 w_{2}+1\right)+f_{2}\left(1-9 w_{1} w_{2}\right)\right]}{\tau f_{2}\left(3 w_{1}+1\right)\left(3 w_{2}+1\right)} \mathcal{E}^{\prime}+\frac{k^{2}\left[\left(3 w_{1}+1\right) f_{2}-4 f_{1}\right]}{3 f_{2}\left(3 w_{2}+1\right)} \mathcal{E} \\
& +k^{2} \tau^{2} \frac{\left(3 w_{1}+1\right)^{2}\left(f_{2}\left(3 w_{1}+1\right)-2 f_{1}\right)}{6 f_{2}\left(3 w_{2}+1\right)} \Phi_{2}+\tau\left(-\frac{2 f_{1}}{f_{2}}+3 w_{1}+1\right) \Phi_{2}^{\prime} \\
& -\frac{\tau\left(3 w_{1}+1\right)\left[f_{2}\left(3 w_{1}+1\right)-2 f_{1}\right]}{f_{2}\left(3 w_{2}+1\right)} \Phi_{1}^{\prime}-k^{2} \tau^{2} \frac{\left(3 w_{1}+1\right)^{2}\left(f_{2}\left(3 w_{1}+1\right)-2 f_{1}\right)}{6 f_{2}\left(3 w_{2}+1\right)} \Phi_{1}=0
\end{aligned}
$$

The quantities $f_{1}$ and $f_{2}$ are defined in (A13). Imposing that all mass terms are positive we get precisely condition (4.14). Notice that for $w_{2}=w_{1}$ the equation for $\mathcal{E}$ reduces to

$$
\mathcal{E}^{\prime \prime}-\frac{6\left(w_{1}-1\right)}{\tau\left(3 w_{1}+1\right)} \mathcal{E}^{\prime}+\frac{k^{2}\left(w_{1}-1\right)}{3 w_{1}+1} \mathcal{E}+\mathcal{F}\left(\Phi_{1}, \Phi_{2}\right)=0,
$$

and the exponential instability is present.

[1] C. de Rham and G. Gabadadze, Phys. Rev. D 82, 044020 (2010).

[2] S. F. Hassan and R. A. Rosen, J. High Energy Phys. 07 (2011) 009.

[3] M. Fierz and W. Pauli, Proc. R. Soc. A 173, 211 (1939).

[4] C. de Rham, G. Gabadadze, and A. J. Tolley, Phys. Rev. Lett. 106, 231101 (2011).

[5] S. F. Hassan and R. A. Rosen, Phys. Rev. Lett. 108, 041101 (2012).

[6] G. D'Amico, C. de Rham, S. Dubovsky, G. Gabadadze, D. Pirtskhalava, and A. J. Tolley, Phys. Rev. D 84, 124046 (2011).

[7] A. E. Gumrukcuoglu, C. Lin, and S. Mukohyama, J. Cosmol. Astropart. Phys. 11 (2011) 030.

[8] N. Khosravi, G. Niz, K. Koyama, and G. Tasinato, J. Cosmol. Astropart. Phys. 08 (2013) 044.

[9] A. De Felice, A. E. Gumrukcuoglu, and S. Mukohyama, Phys. Rev. Lett. 109, 171101 (2012).

[10] A. De Felice, A.E. Gumrukcuoglu, C. Lin, and S. Mukohyama, J. Cosmol. Astropart. Phys. 05 (2013) 035.

[11] N. Arkani-Hamed, H. Georgi, and M. D. Schwartz, Ann. Phys. (Amsterdam) 305, 96 (2003).

[12] C. de Rham, Living Rev. Relativity 17, 7 (2014).

[13] A.DeFelice, A. E. Gumrukcuoglu, C.Lin, and S. Mukohyama, Classical Quantum Gravity 30, 184004 (2013).

[14] V. A. Rubakov, arXiv:hep-th/0407104.
[15] S. L. Dubovsky, J. High Energy Phys. 10 (2004) 076.

[16] D. Comelli, F. Nesti, and L. Pilo, Phys. Rev. D 87, 124021 (2013).

[17] D. Comelli, M. Crisostomi, F. Nesti, and L. Pilo, Phys. Rev. D 86, 101502 (2012).

[18] D. Comelli, F. Nesti, and L. Pilo, J. High Energy Phys. 07 (2013) 161.

[19] D. Comelli, F. Nesti, and L. Pilo, J. Cosmol. Astropart. Phys. 05 (2014) 036.

[20] T. Damour and I. I. Kogan, Phys. Rev. D 66, 104024 (2002).

[21] Z. Berezhiani, D. Comelli, F. Nesti, and L. Pilo, Phys. Rev. Lett. 99, 131101 (2007).

[22] C. J. Isham, A. Salam, and J. A. Strathdee, Phys. Rev. D 3, 867 (1971); A. Salam and J. A. Strathdee, Phys. Rev. D 16, 2668 (1977).

[23] D. Comelli, M. Crisostomi, F. Nesti, and L. Pilo, J. High Energy Phys. 03 (2012) 067.

[24] M. von Strauss, A. Schmidt-May, J. Enander, E. Mortsell, and S. F. Hassan, J. Cosmol. Astropart. Phys. 03 (2012) 042.

[25] M. S. Volkov, J. High Energy Phys. 01 (2012) 035.

[26] D. Comelli, M. Crisostomi, and L. Pilo, J. High Energy Phys. 06 (2012) 085.

[27] Z. Berezhiani, F. Nesti, L. Pilo, and N. Rossi, J. High Energy Phys. 07 (2009) 083.

[28] Z. Berezhiani, L. Pilo, and N. Rossi, Eur. Phys. J. C 70, 305 (2010). 
[29] L. Pilo, Proc. Sci. HEP2011 (2011) 076.

[30] D. Comelli, M. Crisostomi, F. Nesti, and L. Pilo, Phys. Rev. D 85, 024044 (2012).

[31] Z. Berezhiani, D. Comelli, F. Nesti, and L. Pilo, J. High Energy Phys. 07 (2008) 130.

[32] S. F. Hassan and R. A. Rosen, J. High Energy Phys. 02 (2012) 126.

[33] S. F. Hassan, R. A. Rosen, and A. Schmidt-May, J. High Energy Phys. 02 (2012) 026; S. F. Hassan and R. A. Rosen, J. High Energy Phys. 04 (2012) 123.

[34] A. De Felice, T. Nakamura, and T. Tanaka, Prog. Theor. Exp. Phys. 2014, 043E01 (2014).
[35] M. Berg, I. Buchberger, J. Enander, E. Mortsell, and S. Sjors, J. Cosmol. Astropart. Phys. 12 (2012) 021.

[36] Y. Akrami, T. S. Koivisto, and M. Sandstad, J. High Energy Phys. 03 (2013) 099.

[37] F. Knnig and L. Amendola, Phys. Rev. D 90, 044030 (2014).

[38] E. Babichev and M. Crisostomi, Phys. Rev. D 88, 084002 (2013); E. Babichev and Cd. Deffayet, Classical Quantum Gravity 30, 184001 (2013).

[39] A. De Felice, A. E. Gümrükçüoğlu, S. Mukohyama, N. Tanahashi, and T. Tanaka, J. Cosmol. Astropart. Phys. 06 (2014) 037. 